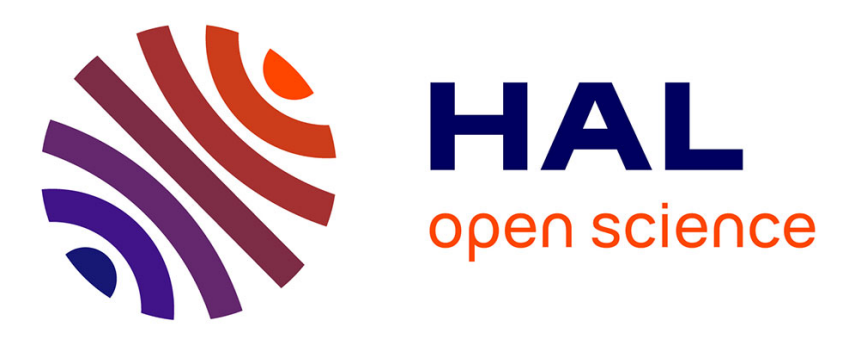

\title{
Forcing-based cut-elimination for Gentzen-style intuitionistic sequent calculus
}

Hugo Herbelin, Gyesik Lee

\section{To cite this version:}

Hugo Herbelin, Gyesik Lee. Forcing-based cut-elimination for Gentzen-style intuitionistic sequent calculus. Workshop on Logic, Language, Information and Computation, Jun 2009, Tokyo, Japan. pp.209-217. inria-00381554

\section{HAL Id: inria-00381554 \\ https://hal.inria.fr/inria-00381554}

Submitted on 5 May 2009

HAL is a multi-disciplinary open access archive for the deposit and dissemination of scientific research documents, whether they are published or not. The documents may come from teaching and research institutions in France or abroad, or from public or private research centers.
L'archive ouverte pluridisciplinaire HAL, est destinée au dépôt et à la diffusion de documents scientifiques de niveau recherche, publiés ou non, émanant des établissements d'enseignement et de recherche français ou étrangers, des laboratoires publics ou privés. 


\title{
Forcing-based cut-elimination for Gentzen-style intuitionistic sequent calculus
}

\author{
Hugo Herbelin ${ }^{1}$ and Gyesik Lee ${ }^{2}$ \\ 1 INRIA \& PPS, Paris Université 7 \\ Paris, France \\ Hugo.Herbelin@inria.fr \\ 2 ROSAEC center, Seoul National University \\ Seoul, Korea \\ gslee@ropas.snu.ac.kr
}

\begin{abstract}
We give a simple intuitionistic completeness proof of Kripke semantics with constant domain for intuitionistic logic with implication and universal quantification. We use a cut-free intuitionistic sequent calculus as formal system and by combining soundness with completeness, we obtain an executable cut-elimination procedure. The proof, which has been formalised in the Coq proof assistant, easily extends to the case of the absurdity connective using Kripke models with exploding nodes.
\end{abstract}

Keywords: Intuitionistic Gentzen-style sequent calculus, Kripke semantics, completeness, cut-elimination

\section{Introduction}

The intuitionistic completeness proofs for intuitionistic full first-order predicate logic given by Veldman [1] and Friedman [2, Chapter 13] use nonstandard Kripke model and Beth model, respectively (the false formula may be forced at some nodes). Both the proof of Veldman and that of Friedman work by building a model made of infinite contexts. Especially, they had to deal with language extensions and work with spreads in order to meet some closure conditions for disjunction and existential quantification:

- $\Gamma \vdash A \vee B$ implies $\Gamma \vdash A$ or $\Gamma \vdash B$.

- $\Gamma \vdash \exists x A(x)$ implies $\Gamma \vdash A(c)$ for some constant $c$.

Note however that this is not only the case for intuitionistic proofs, but also the case for a classical, Henkin-type proof given in Troelstra and van Dalen [2, Chapter 2].

On the other hand, C. Coquand 3] shows that an intuitionistic proof for intuitionistic propositional logic with implication as a sole logical symbol can be obtained in a much simpler way by building a universal model made of finite contexts of formulae. She gave a mechanised proof of the completeness proof and 
even got a cut-elimination proof by using some interpreter and inversion functions, a method called "normalisation by evaluation" in general, cf. 4]. They correspond to the soundness and the completeness, respectively, of the propositional logic with implication as sole connective w.r.t. Kripke semantics. The completeness result there is strong in two ways: in the traditional sense that it holds in arbitrary contexts (see 2]) and in a sense (due to Okada 5) that it builds normal proofs. In this paper, we extend C. Coquand's idea to the intuitionistic first-order predicate logic with implication and universal quantification as logical symbols.

The predicate system used here is a Gentzen-style sequent calculus. The advantage is that the notion of normal proofs is easy to define: one just has to remove the cut rule. More precisely, the calculus we consider is the intuitionistic restriction LJT of a sequent calculus named LKT that Danos et al [6] derived from an analytical decomposition of Gentzen's LK within Girard's Linear Logic [7]. LKT is a constrained variant of LK. Its main property is the bijective correspondence between its set of cut-free proofs and the set of normal form $\AA^{3}$ of classical natural deduction [8. LJT itself is a constrained variant of LJ and its main property is the bijective correspondence between its set of cut-free proofs and the set of normal forms of natural deduction 910. By choosing LJT and its cut-free variant as our reference calculus, we emphasise that our completeness theorem builds not any arbitrary proofs but cut-free ones in a subset of LJ which bijectively maps to normal natural deduction proofs (and hence to normal $\lambda$-terms).

We show that the strong completeness holds both for the system with or without $\left(\perp_{i}\right)$. In case of with $\left(\perp_{i}\right)$, we adopt Veldman's modified Kripke semantics. Both proofs are intuitionistic and almost the same. Therefore, we can get a very simple cut-elimination proofs as a by-product at the end. A main difference compared with Veldman's or Friedman's proof is that we deal with contexts made of formulae, not just of sentences, therefore we need to handle substitutions.

The Kripke models we are considering are Kripke models with constant domain, i.e. with the domain function $D$ being the same for every world:

$$
D=D(w) \text { for all } w \text {. }
$$

As a consequence, in the case of universal quantification, considering of all possible future worlds is not necessary and the following simpler definition

$$
w \Vdash \forall x A(x) \text { iff, for all } d \in D, w \Vdash A(d) .
$$

gets equivalent, by monotonicity of forcing and invariance of the domain, to the standard definition:

$$
w \Vdash \forall x A(x) \text { iff, for all } w^{\prime} \geq w \text { and for all } d \in D, w^{\prime} \Vdash A(d) \text {. }
$$

\footnotetext{
3 To be precise: normal forms along a call-by-name reduction semantics.
} 
This paper is organised as follows. We first prove the soundness of the Kripke semantics with respect to cut-free LJT. Then give a intuitionistic strong completeness proof which results in the intuitionistic completeness proof. Finally, an intuitionistic cut-elimination process is described.

\section{The sequent calculus LJT}

Let $\mathcal{L}=\mathcal{L}(\mathbb{C}, \mathbb{F}, \mathbb{P})$ be a first-order language with an infinite set $\mathbb{C}$ of individual constants, among them a distinguished constant $c_{0}$, a non-empty set $\mathbb{F}$ of functions, and a non-empty set $\mathbb{P}$ of predicates. The logical symbols are the implication $\rightarrow$ and the universal quantification $\forall$. We assume furthermore that there are countably many free variables. Terms, formulae and sentences are defined in the usual way. We follow the convention that $A(x)$ denotes the formula $A$ where the variable $x$ might appear. Any two formulae are considered identical when they are different only in names for bound variables. A variable or a constant is called fresh in a formula $A$ or a context $\Gamma$ when it does not occur free or at all, respectively.

Definition 1 (Simultaneous substitutions). Let $\rho$ be a function from finite set of variables to the set of terms.

1. Given a term $t, t[\cdot \backslash \rho]$ is inductively defined:

$$
\begin{aligned}
& -x[. \backslash \rho]= \begin{cases}\rho(x) & \text { if } x \in \operatorname{dom}(\rho), \\
x & \text { otherwise. }\end{cases} \\
& -c[\cdot \backslash \rho]=c \text { for any } c \in \mathbb{C} . \\
& -\left(f t_{1} \cdots t_{n}\right)[\cdot \backslash \rho]=f\left(t_{1}[\cdot \backslash \rho]\right) \cdots\left(t_{n}[. \backslash \rho]\right) .
\end{aligned}
$$

2. Given a formula $A, A[\cdot \backslash \rho]$ is inductively defined:

$$
\begin{aligned}
& -\left(P t_{1} \cdots t_{n}\right)[\cdot \backslash \rho]=P\left(t_{1}[. \backslash \rho]\right) \cdots\left(t_{n}[. \backslash \rho]\right) . \\
& -(A \rightarrow B)[\cdot \backslash \rho]=(A[\cdot \backslash \rho] \rightarrow(B[\cdot \backslash \rho]) .
\end{aligned}
$$$$
-(\forall x A)[\cdot \backslash \rho]=\forall x\left(A\left[\cdot \backslash \rho^{-x}\right]\right) \text {. }
$$

Here $\rho^{-x}$ denotes the function obtained from $\rho$ with $\operatorname{dom}\left(\rho^{-x}\right)=\operatorname{dom}(\rho) \backslash\{x\}$, i.e., if $y \in \operatorname{dom}(\rho)$ and $x \neq y$, then $\rho^{-x}(y)=\rho(y)$ and undefined otherwise. We also take care of variable capture by changing bound variables when necessary.

Given a formula $A(x)$, we use $A_{x}(t)$ or $A[x \backslash t]$ for $A[\cdot \backslash \rho]$ where $\rho=\{(x, t)\}$. We also consider substitution of a term $t$ for a constant $c$ in a similar way and use the notation $A_{c}(t)$.

The Gentzen-style sequence calculus LJT is obtained from the intuitionistic sequent calculus LJ by restricting the use of the left introduction rules of the implication and the universal quantification. See Table 1 for the cut-free fragment. In that way, one can get a one-to-one correspondence between cut-free proofs in LJT and normal terms in $\lambda$-style calculus.

In LJT, a sequent has one of the forms $\Gamma ; A \vdash C$ or $\Gamma \vdash C$, where $\Gamma$ is a list of formulae. That is, the location of a formula occurring multiple times is important. The right side of ";" in the antecedence is called stoup . $\Gamma, \Gamma^{\prime}, \Delta, \ldots$ 


\begin{tabular}{|cc|}
\hline$\frac{\Gamma ; A \vdash C \quad A \in \Gamma}{\Gamma ; A \vdash A}(A x)$ & $\frac{A, \Gamma \vdash B}{\Gamma \vdash C}\left(\rightarrow_{r}\right)$ \\
$\frac{\Gamma \vdash A \quad \Gamma ; B \vdash C}{\Gamma ; A \rightarrow B \vdash C}(\rightarrow \ell)$ & $\frac{\Gamma \vdash A(x) \quad x \text { fresh in } \Gamma}{\Gamma \vdash \forall x A}\left(\forall_{r}\right)$ \\
$\frac{\Gamma ; A_{x}(t) \vdash C}{\Gamma ; \forall x A(x) \vdash C}\left(\forall_{\ell}\right)$ & $\frac{\Gamma+B \text { i }}{\Gamma+A}$ \\
\hline
\end{tabular}

Table 1. Cut-free LJT

vary over lists of formulae. We write $A \in \Gamma$ when $A$ occurs in $\Gamma . \Gamma \sqsubseteq \Delta$ denotes that, for all $A, A \in \Gamma$ implies $A \in \Delta . \Gamma_{c}(t)$ is obtained from $\Gamma$ by replacing each formula $A$ with $A_{c}(t)$.

Lemma 2 (Weakening and Exchange). Let $A, C$ be formulae and $\Gamma, \Gamma^{\prime}$ two contexts such that $\Gamma \sqsubseteq \Gamma^{\prime}$.

1. $\Gamma \vdash C$ implies $\Gamma^{\prime} \vdash C$.

2. $\Gamma ; A \vdash C$ implies $\Gamma^{\prime} ; A \vdash C$.

Proof. One can easily prove both claims by a simultaneous induction on the deduction.

Lemma 3. Let $\Gamma$ be a context, $A, C$ formulae, and c a constant.

1. $\Gamma \vdash C$ implies $\Gamma_{c}(y) \vdash C_{c}(y)$ for any variable $y$ which is not bound in $\Gamma, A$.

2. $\Gamma ; A \vdash C$ implies $\Gamma_{c}(y) ; A_{c}(y) \vdash C_{c}(y)$ for any variable $y$ which is not bound in $\Gamma, A, C$.

Proof. By a simple simultaneous induction on deduction.

The following lemma says that a fresh constant is as good as a fresh variable and will play an important role in the proof of the strong completeness.

Lemma 4. Given a context $\Gamma$, a formula $A(x)$, and a constant $c$ fresh in $\Gamma$ and $A(x), \Gamma \vdash A_{x}(c)$ implies $\Gamma \vdash A_{x}(y)$ for any variable $y$ which is not bound in $\Gamma, A$.

Proof. It follows directly from the lemma just before.

\section{$3 \quad$ Kripke semantics}

Kripke semantics was created in the late 1950s and early 1960s by Saul Kripke [1112. It was first made for modal logic, and later adapted to intuitionistic logic and other non-classical systems. In this section we discuss Kripke models for the first-order predicate logic with implication and universal quantification as sole logical symbols and their connection with intuitionistic validity. 
Definition 5. A Kripke model is a quadruple $\mathcal{K}=(\mathcal{W}, \leq, \Vdash, \mathcal{D}, V), \mathcal{W}$ inhabited, such that

1. $(\mathcal{W}, \leq)$ is a partially ordered set.

2. $\mathcal{D}$ is an inhabited set, called the domain of $\mathcal{K}$.

3. Let the language be extended with constant symbols for each element of $\mathcal{D}$. Then $\Vdash$ is a relation between $\mathcal{W}$ and the set of prime sentences in the extended language such that

$$
\left(w \leq w^{\prime} \wedge w \Vdash P d_{1} \cdots d_{n}\right) \quad \Rightarrow \quad w^{\prime} \Vdash P d_{1} \cdots d_{n}
$$

where $w, w^{\prime} \in \mathcal{W}, P \in \mathbb{P}, d_{1}, \ldots, d_{n} \in \mathcal{D}$, and $n=\operatorname{arity}(P)$.

4. $V$ is a function such that

- $V(c) \in \mathcal{D}$ for all $c \in \mathbb{C}$.

- $V(f): \mathcal{D}^{\text {arity }(f)} \rightarrow \mathcal{D}$ for all $f \in \mathbb{F}$.

An association $\rho$ based on $\mathcal{K}$ is a function from a finite set of variables to $\mathcal{D}$. Given an association $\rho$, each term has an interpretation in $\mathcal{D}$ :

$-x[\rho]= \begin{cases}\rho(x) & \text { if } x \in \operatorname{dom}(\rho), \\ V\left(c_{0}\right) & \text { otherwise. }\end{cases}$

$-c[\rho]=V(c)$ for any $c \in \mathbb{C}$.

- $\left(f t_{1} \cdots t_{\ell}\right)[\rho]=V(f)\left(t_{1}[\rho]\right) \cdots\left(t_{\ell}[\rho]\right)$.

The forcing relation is then inductively extended by the forthcoming clauses to all $\mathcal{L}$-formulae.

$-w \Vdash\left(P t_{1} \cdots t_{n}\right)[\rho]$ iff $w \Vdash P\left(t_{1}[\rho]\right) \cdots\left(t_{n}[\rho]\right)$.

$-w \Vdash(A \rightarrow B)[\rho]$ iff, for all $w^{\prime} \geq w$, if $w^{\prime} \Vdash A[\rho]$, then $w^{\prime} \Vdash B[\rho]$.

$-w \Vdash(\forall x A)[\rho]$ iff, for all $d \in D, w \Vdash A[\rho(x \mapsto d)]$.

Here $\rho(x \mapsto d)$ denotes the association $\rho^{\prime}$ such that $\rho^{\prime}(y)=\rho(y)$ if $y \neq x$ and $\rho^{\prime}(x)=d$. The definition of forcing is extended to contexts as follows:

$$
w \Vdash \Gamma[\rho] \text { iff } w \Vdash A[\rho] \text { for all } A \in \Gamma,
$$

Remark 6. There are two points to be mentioned.

1. The forcing relation is upward monotone, i.e., if $w \leq w^{\prime}$ and $w \Vdash A[\rho]$, then $w^{\prime} \Vdash A[\rho]$.

2. As mentioned in the introduction, the forcing definition at the universal quantification case is much simpler than the usual definition, where the domain depends on worlds:

$w \Vdash(\forall x A(x))[\rho]$ iff, for all $w^{\prime} \geq w$ and $d \in D(w), w^{\prime} \Vdash A[\rho(x \rightarrow d)]$.

Indeed, they are "functionally equivalent" in the sense that soundness and completeness hold in both cases.

We consider a formulation of Kripke semantics for sequent calculus built on two kinds of judgements $\Gamma \vdash C$ and $\Gamma ; A \vdash C$. 
Theorem 7 (Soundness). We have the following soundness.

1. if $\Gamma \vdash C$ then, for all $w$ and $\rho, w \Vdash \Gamma[\rho]$ implies $w \Vdash C[\rho]$.

2. if $\Gamma ; A \vdash C$ then, for all $w$ and $\rho$, $(w \Vdash \Gamma[\rho]$ and $w \Vdash A[\rho])$ implies $w \Vdash C[\rho]$.

Proof. By a simultaneous induction on the deduction.

\section{Completeness}

In this section we present a constructive completeness proof by constructing a simple universal model. First we construct a universal model for which a strong completeness holds.

Definition 8 (Universal Kripke model). The universal Kripke model $\mathcal{U}=$ $\left(\mathcal{W}_{u}, \sqsubseteq, \Vdash_{u}, \mathcal{D}_{u}, V_{u}\right)$ is defined as follows:

- $\mathcal{W}_{u}$ is the set of all contexts.

- $\sqsubseteq$ denotes the sub-context relation, i.e., $\Gamma \sqsubseteq \Gamma^{\prime}$ holds when, for all $A \in \Gamma$, $A \in \Gamma^{\prime}$.

- $\mathcal{D}_{u}$ consists of all closed terms.

- $V_{u}(c)=c$ for all $c \in \mathbb{C}$, and $V_{u}(f)\left(t_{1}, \ldots, t_{\ell}\right)=f t_{1} \cdots t_{\ell}$ for all $f \in \mathbb{F}$ and $t_{1}, \ldots, t_{\ell} \in \mathcal{D}_{u}^{\operatorname{arity}(f)}$

$-\Gamma \Vdash P t_{1} \cdots t_{\ell}$ if $\Gamma \vdash P t_{1} \cdots t_{\ell}$. It is obvious that $\Gamma \sqsubseteq \Gamma^{\prime}$ and $\Gamma \vdash P t_{1} \cdots t_{\ell}$ imply $\Gamma^{\prime} \vdash t_{1} \cdots t_{\ell}$.

Theorem 9 (Strong Completeness). Let $\Gamma$ be a context of sentences, $A$ a formula, and $\rho$ an association based on $\mathcal{K}_{u}$ such that $F V(A) \subseteq \operatorname{dom}(\rho)$. Then

1. If $\Gamma \Vdash A[\rho]$ then $\Gamma \vdash A[\cdot \backslash \rho]$.

2. If, for all formula $C$ and context $\Gamma^{\prime}$ such that $\Gamma \sqsubseteq \Gamma^{\prime}, \Gamma^{\prime} ; A[. \backslash \rho] \vdash C$ implies $\Gamma^{\prime} \vdash C$, then it holds that $\Gamma \Vdash A[\rho]$.

Proof. Given the assumptions we prove both claims by a simultaneous induction on the complexity of $A$. Note first that $t[\rho]=t[. \backslash \rho]$ for any term $t$ occurring in $A$ since $F V(A) \subseteq \operatorname{dom}(\rho)$.

1. case: $A$ is a prime formula. Then the first claim is obvious. For the second claim take just $\Gamma^{\prime}:=\Gamma$ and $C:=A[\cdot \backslash \rho]$.

2. case: $A=A_{1} \rightarrow A_{2}$.

- Assume $\Gamma \Vdash\left(A_{1} \rightarrow A_{2}\right)[\rho]$. To show $\Gamma \vdash A_{1}[\cdot \backslash \rho] \rightarrow A_{2}[\cdot \backslash \rho]$, it suffices to prove that $A_{1}[\cdot \backslash \rho], \Gamma \vdash A_{2}[\cdot \backslash \rho]$. Note that the i.h. on $A_{1}$ for the second claim implies that $A_{1}[\cdot \backslash \rho], \Gamma \Vdash A_{2}[\rho]$. Indeed, the premise of the second claim holds trivially for any $\Gamma^{\prime}$ such that $A_{1}[\cdot \backslash \rho], \Gamma \sqsubseteq \Gamma^{\prime}$. This in turn implies that $A_{1}[\cdot \backslash \rho], \Gamma \Vdash A_{2}[\rho]$ by the assumption. Then the i.h. on $A_{2}$ for the first claim leads to the goal. 
- Assume for all formula $C$ and context $\Gamma^{\prime}$ such that $\Gamma \sqsubseteq \Gamma^{\prime}, \Gamma^{\prime} ; A_{1}[\cdot \backslash \rho] \rightarrow$ $A_{2}[\cdot \vee \rho] \vdash C$ implies $\Gamma^{\prime} \vdash C$. Assume furthermore that $\Gamma \sqsubseteq \Delta$ and $\Delta \Vdash A_{1}[\rho]$. Then it remains to show $\Delta \Vdash A_{2}[\rho]$. For that we apply the i.h. on $A_{2}$ for the second claim. Let $C$ be a formula and $\Delta^{\prime}$ a context such that $\Delta \sqsubseteq \Delta^{\prime}$, assume $\Delta^{\prime} ; A_{2}[\cdot \backslash \rho] \vdash C$. Note that $\Delta \vdash A_{1}[\cdot \backslash \rho]$ by i.h. on $A_{1}$ for the first claim, hence $\Delta^{\prime} \vdash A_{1}[. \backslash \rho]$ by the Weakening Lemma 2 . By applying $(\rightarrow \ell)$ we get $\Delta^{\prime} ;\left(A_{1} \rightarrow A_{2}\right)[\cdot \backslash \rho] \vdash C$, so $\Delta^{\prime} \vdash C$ holds by the assumption.

3. case: $A=\forall x B(x)$.

- Assume $\Gamma \Vdash(\forall x B)[\rho]$, i.e., for all closed term $t, \Gamma \Vdash B[\rho(x \mapsto t)]$. To show $\Gamma \vdash \forall x\left(B\left[. \backslash \rho^{-x}\right]\right)$, we need to prove that $\Gamma \vdash\left(B\left[\cdot \backslash \rho^{-x}\right]\right)[x \backslash y]$ for some variable $y$ fresh in $\Gamma$ and $B\left[. \backslash \rho^{-x}\right]$. For this we show $\Gamma \vdash\left(B\left[. \backslash \rho^{-x}\right]\right)[x \backslash c]$ for some constant $c$ and apply Lemma 4 . Let $c$ be a constant fresh in $\Gamma$ and $B\left[\cdot \backslash \rho^{-x}\right]$. Note first that

$$
\left(B\left[\cdot \backslash \rho^{-x}\right]\right)[x \backslash c]=B\left[\cdot \backslash \rho^{-x}(x \mapsto c)\right]=B[\cdot \backslash \rho(x \mapsto c)]
$$

since the values of $\rho$ are closed terms. However, $\Gamma \vdash B[\cdot \backslash \rho(x \mapsto c)]$ follows from the i.h. on $B$ for the first claim 4

- Assume for all formula $C$ and context $\Gamma^{\prime}$ such that $\Gamma \sqsubseteq \Gamma^{\prime}$, it holds that $\Gamma^{\prime} ; \forall x\left(B\left[\cdot \backslash \rho^{-x}\right]\right) \vdash C$ implies $\Gamma^{\prime} \vdash C$. Given a closed term $t$, we have to show that $\Gamma \Vdash B[\rho(x \mapsto t)]$. In order to apply the i.h. on $B$ for the second claim assume furthermore that a formula $C$ and a context $\Gamma^{\prime}$ are given such that $\Gamma \sqsubseteq \Gamma^{\prime}$ and $\Gamma^{\prime} ; B[. \backslash \rho(x \mapsto t)] \vdash C$. Note that $B[. \backslash \rho(x \mapsto t)]=$ $B\left[. \backslash \rho^{-x}\right][x \backslash t]$ since only closed terms are substituted. Therefore, it holds that $\Gamma^{\prime} ; \forall x\left(B\left[. \backslash \rho^{-x}\right]\right) \vdash C$. Then by the main assumption, $\Gamma^{\prime} \vdash C$. The i.h. implies $\Gamma \Vdash B[\rho(x \mapsto t)]$.

Corollary 10. For any context $\Gamma$ of sentences, $\Gamma \Vdash \Gamma$.

Proof. The second claim of the strong completeness and the rule (Contr) implies that $\Gamma \Vdash A$ for any $A \in \Gamma$.

Theorem 11 (Completeness). Let $\Gamma$ be a context of sentences and $A$ a sentence. If for all Kripke model $\mathcal{K}$ and a world $w$ in $\mathcal{K}, w \Vdash \Gamma$ implies $w \Vdash A$, then $\Gamma \vdash A$.

Proof. It follows from the Strong Completeness and the fact that $\Gamma \Vdash \Gamma$, i.e., $\Gamma \vdash A$ iff $\Gamma \Vdash A$.

Corollary 12. In the intuitionistic predicate logic with implication and universal quantification as sole connectives, the Kripke semantics with constant domain is functionally equivalent to the usual Kripke semantics where the domain depends on worlds in the sense that soundness and completeness hold in both cases.

\footnotetext{
${ }^{4}$ One can see here that we don't need any quantification in the definition of $w \Vdash$ $\forall x A(x)$.
} 
Proof. The exactly same proofs for soundness and completeness hold with the usual Kripke models.

Remark 13. We furthermore believe that, in the first-order predicate language with $\rightarrow, \wedge$ and $\forall$ as sole connectives, the usual Kripke semantics is equivalent to the Kripke semantics with constant domain in the sense that each usual Kripke model can be transformed into an equivalent Kripke model with a constant domain.

Remark 14. The completeness above easily extends to the case of the absurdity connective

$$
\frac{\Gamma \vdash \perp}{\Gamma \vdash C}\left(\perp_{i}\right)
$$

using modified Kripke models with exploding nodes à la Veldman.

A modified Kripke model $\mathcal{K}=(\mathcal{W}, \leq, \Vdash, \mathcal{D}, V)$ is defined as the (unmodified) Kripke model, but with one change:

$-w \Vdash\left(P t_{1} \cdots t_{n}\right)[\rho]$ iff $\left(w \Vdash P\left(t_{1}[\rho]\right) \cdots\left(t_{n}[\rho]\right)\right.$ or $\left.w \Vdash \perp\right)$.

The universal Kripke model is defined in the same way as before, but with the following additional clause:

$-\Gamma \Vdash \perp$ iff $\Gamma \vdash \perp$.

Then nothing new is involved in the proof of completeness, the construction proceeds as before. Note only that $\Gamma \vdash \perp$ implies $\Gamma \vdash A$ for any formula $A$.

On the other hand, if we include the absurdity rule and want to stick to the (unmodified) Kripke semantics, we have to give up constructiveness in the proof above. This is because we have to deal only with consistent context $\Gamma$, i.e., $\Gamma \nvdash \perp$. However, it is in general undecidable to check if a context is consistent or not. In the implication case of the strong completeness, we had to make case distinction between $A_{1}[\cdot \backslash \rho], \Gamma \vdash \perp$ or not. This maybe is not so surprising because, in the full first-order predicate logic, an intuitionistic completeness proof entails Markov's Principle, a non-intuitionistic principle, see Kreisel [13].

\section{Cut admissibility}

In this section we consider only sentences and contexts of sentences. Then the cut-rule is admissible with/without $\left(\perp_{i}\right)$.

Theorem 15 (Cut admissibility). $\Gamma \vdash A$ and $\Gamma ; A \vdash B$ imply $\Gamma \vdash B$.

Proof. By the Strong Completeness it suffices to show $\Gamma \Vdash B$. But this follows from the Soundness applied on the two assumptions. Note also that $\vdash$ denotes a cut-free system. 


\section{Conclusion}

We extended C. Coquand's proof of soundness and completeness for implicative natural deduction w.r.t. Kripke semantics 3 to the case of predicate logic with implication and universal quantification. We could show that omitting disjunction and existential quantification from the intuitionistic first-order predicate logic results, as it was the case for C. Coquand, in a significantly simple, intuitionistic completeness proof with respect to (also simplified) Kripke semantics.

The fact that all of the proofs given in this paper are intuitionistic has been verified in the proof assistant Coq, cf. 14. Indeed, the whole work is formalised, so that we can get a mechanical process producing cut-free proofs. The formalisation is performed using cofinite quantification for fresh variables and a locally named approach with two kinds of names for variables, one for free variables and the other for binders. The formalisation is publicly available from the web page of the first author, directory code/kripke.

\section{References}

1. Veldman, W.: An intuitionistic completeness theorem for intuitionistic predicate logic. J. Symb. Log. 41(1) (1976) 159-166

2. Troelstra, A.S., van Dalen, D.: Constructivism in Mathematics: An Introduction I and II. Volume 121, 123 of Studies in Logic and the Foundations of Mathematics. North-Holland (1988)

3. Coquand, C.: From Semantics to Rules: A Machine Assisted Analysis. In: CSL '93. Volume 832 of Lecture Notes in Computer Science., Springer (1993) 91-105

4. Berger, U., Eberl, M., Schwichtenberg, H.: Normalisation by Evaluation. In: Prospects for Hardware Foundations. Volume 1546 of Lecture Notes in Computer Science., Springer (1998) 117-137

5. Okada, M.: A uniform semantic proof for cut-elimination and completeness of various first and higher order logics. Theor. Comput. Sci. 281(1-2) (2002) 471-498

6. Danos, V., Joinet, J.B., Schellinx, H.: LKQ and LKT: sequent calculi for second order logic based upon dual linear decompositions of the classical implication. In: Advances in Linear Logic. Volume 222. Cambridge University Press (1995) 211-224

7. Girard, J.Y.: Linear logic. Theoretical Computer Science 50 (1987) 1-102

8. Parigot, M.: Lambda-mu-calculus: An algorithmic interpretation of classical natural deduction. In: Logic Programming and Automated Reasoning: International Conference LPAR '92 Proceedings, St. Petersburg, Russia, Springer-Verlag (1992) 190-201

9. Herbelin, H.: A Lambda-Calculus Structure Isomorphic to Gentzen-Style Sequent Calculus Structure. In: CSL '94. Volume 933 of Lecture Notes in Computer Science., Springer (1994) 61-75

10. Herbelin, H.: Séquents qu'on calcule: de l'interprétation du calcul des séquents comme calcul de $\lambda$-termes et comme calcul de stratégies gagnantes. Ph.D. thesis, Université Paris 7 (Jan. 1995)

11. Kripke, S.: A Completeness Theorem in Modal Logic. J. Symb. Log. 24(1) (1959) $1-14$

12. Kripke, S.: Semantical considerations on modal and intuitionistic logic. Acta Philos. Fennica 16 (1963) 83-94 
13. Kreisel, G.: On Weak Completeness of Intuitionistic Predicate Logic. J. Symb. Log. 27(2) (1962) 139-158

14. Bertot, Y., Castéran, P.: Interactive Theorem Proving and Program Development (Coq'Art: The Calculus of Inductive Constructions). Volume XXV of EATCS Texts in Theoretical Computer Science. Springer-Verlag (2004) 